\title{
Erratum to: Dynamic BFECC Characteristic Mapping method for fluid simulations
}

\author{
Xiaosheng Li • Le Liu • Wen Wu • Xuehui Liu • \\ Enhua Wu
}

Published online: 24 May 2014

(C) Springer-Verlag Berlin Heidelberg 2014

\section{Erratum to: Vis Comput}

DOI 10.1007/s00371-014-0969-7

The original version of this article unfortunately contained a mistake. The sequence of Figs. 1-9 was incorrect. The corrected sequence of all figures (Figs. 1, 2, 3, 4, 5, 6, 7, 8, $9,10)$ is given below.
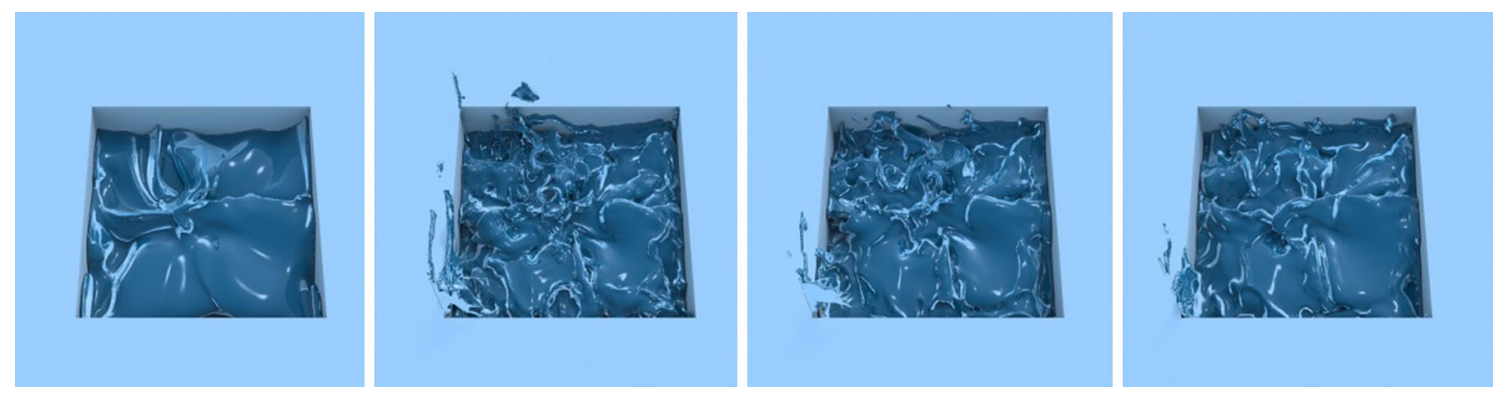

Fig. 1 Comparison of different values of $\epsilon$. From left to right, the first one is semi-Lagrangian advection while the others used $\epsilon$ of $\epsilon x, 3 \Delta x$ and $5 \Delta x$ for DBCM method. Smaller $\epsilon$ led to more detailed and turbulent surface. $\epsilon$ can be used for controlling the details. As level set was

The online version of the original article can be found under doi:10.1007/s00371-014-0969-7.

X. Li (ه) · L. Liu · X. Liu · E. Wu

State Key Laboratory of Computer Science, Institute of Software,

Chinese Academy of Sciences, Beijing, China

e-mail: lixs@ios.ac.cn

X. Li • L. Liu

University of Chinese Academy of Sciences, Beijing, China

W. Wu $\cdot$ E. Wu

University of Macau, Macao, China used for identifying the fluid domain, the underlying simulations could be slightly different with different values of $\epsilon$. Even with high tolerance value, the result was still much better than that of semi-Lagrangian advection 

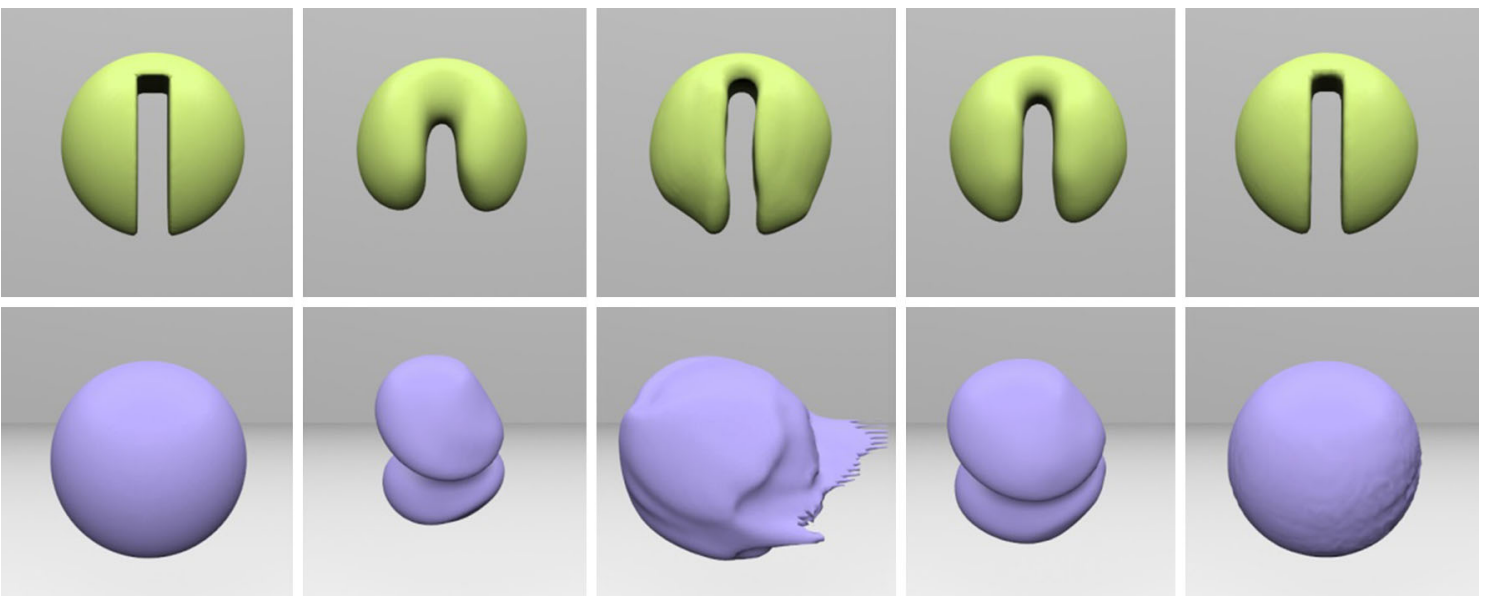

Fig. 2 Results of Zalesak' disk (top) and Enright's sphere (bottom) after one full cycle of rotation and deformation under different advection schemes (start from the second column): 1. Semi-Lagrangian advec- tion; 2. BFECC advection; 3. Linear CM method; 4. DBCM method. The leftmost column is the initial contour. All resolutions used were $128^{3}$
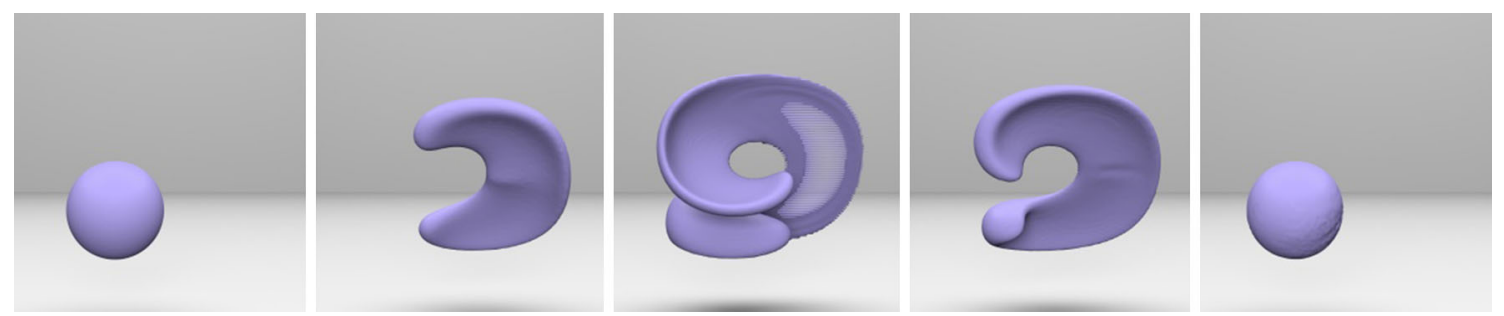

Fig. 3 Enright test of our method with a resolution of $128^{3}$. Note that in the extreme deformation, some details were lost and holes appeared due to the limitation of the resolution

Fig. 4 Two simple 2D smoke

simulations using

semi-Lagrangian advection, semi-Lagrangian CM method and our method (from left to right) on a $256^{2}$ grid. Using BFECC advection for mapping function captures the most details and the result is less diffusive than the other two methods
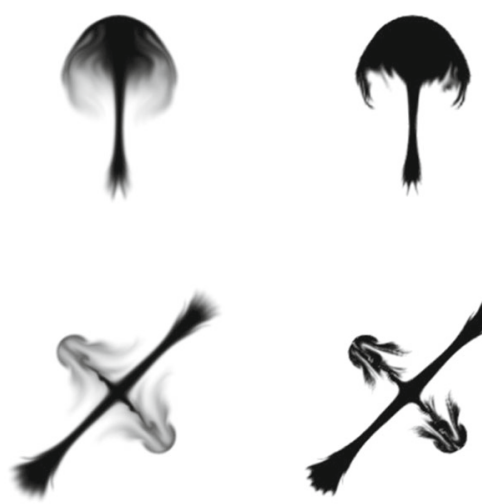

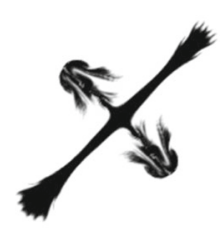

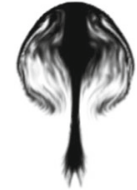

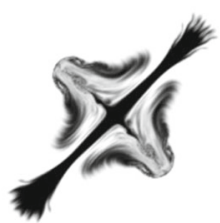



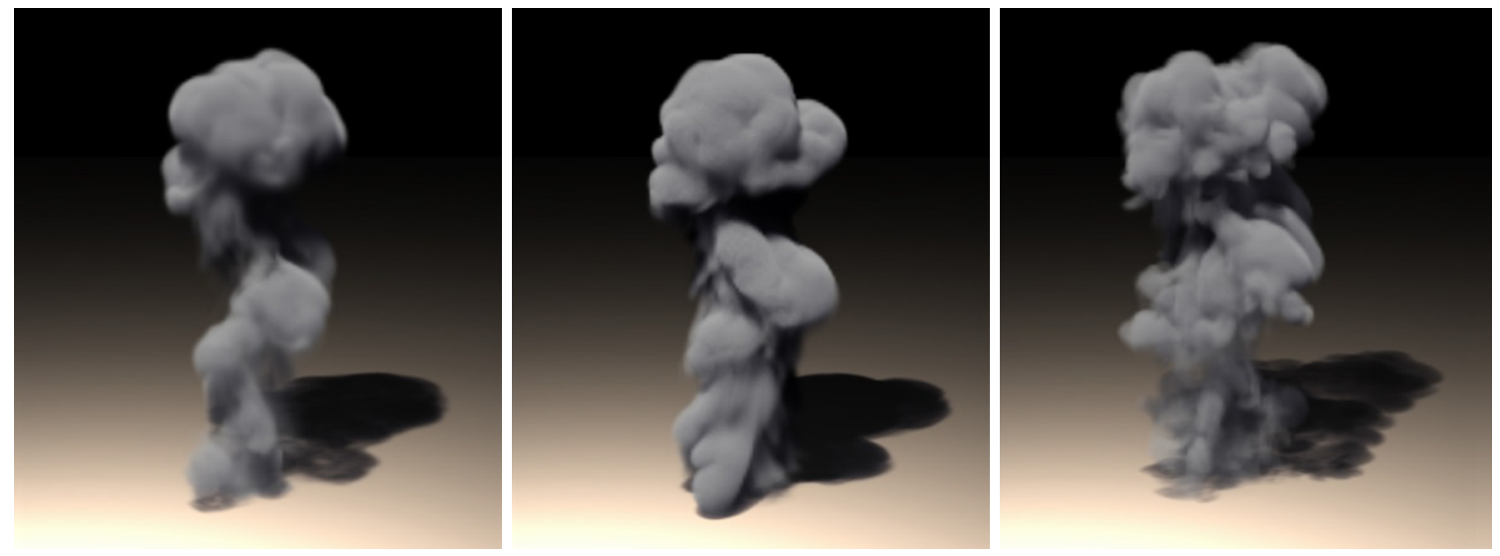

Fig. 5 Snapshots of 3D smoke simulation with linear semi-Lagrangian advection, linear CM method, DBCM method. DBCM produced a result with the most details and details of thin smoke sheets were clearly cap-
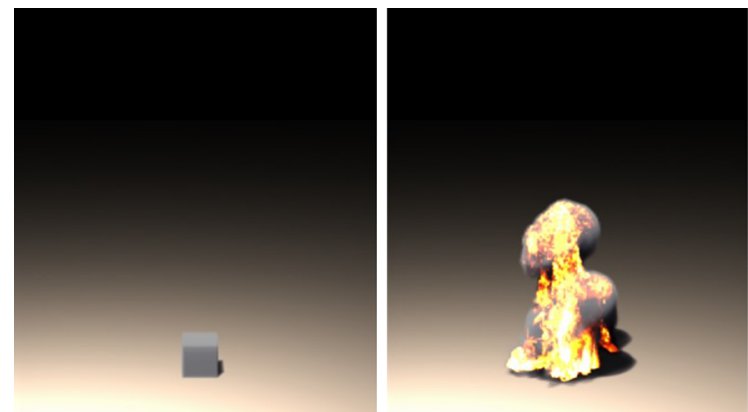

Fig. 6 Smoke simulation with fire simulation using DBCM method. Fuel and temperature field were used to simulate the fire. DBCM advected the density, fuel and temperature field simultaneously with
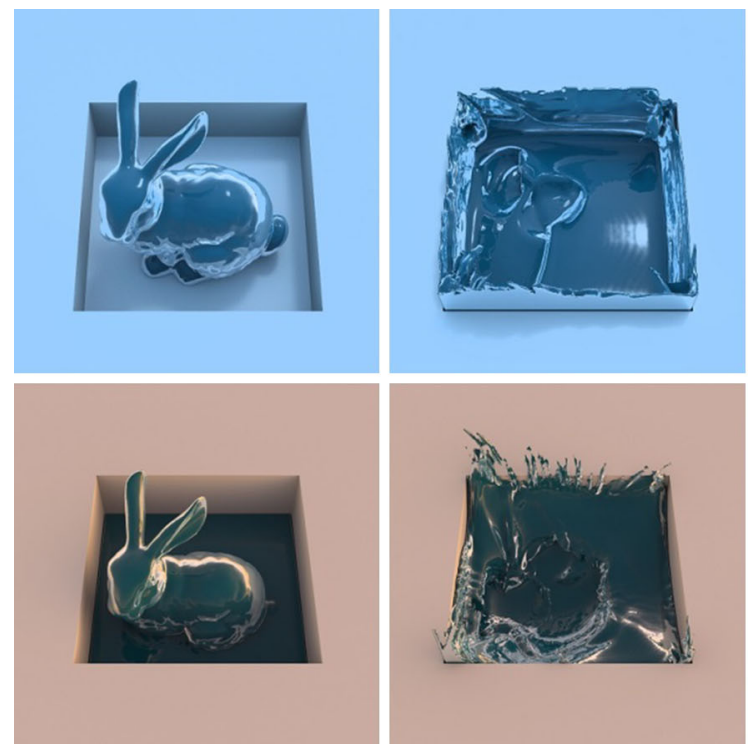

Fig. 7 Semi-Lagrangian water simulation using our method for advection of the level set. Our method is capable of capturing small details of the water. The resolution of base fluid simulation was $128^{3} . N_{c}$ was tured. Resolution of base fluid simulation was $128 \times 256 \times 128 . N_{c}$ was set to 256 for $\chi$ and $N_{f}$ was set to 256 for $\chi^{R}$ for DBCM method
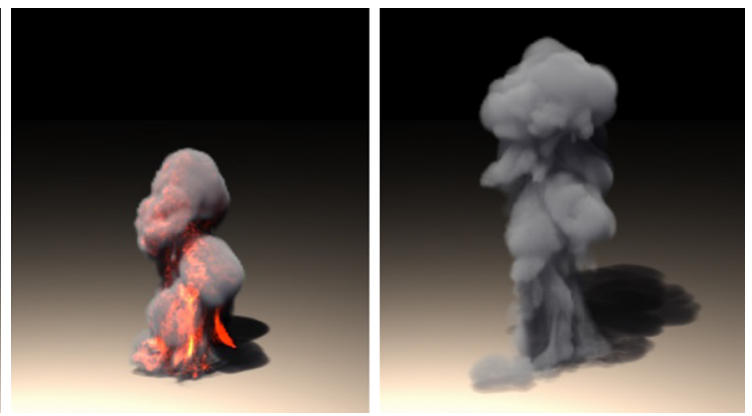

the same mapping function and captured rich details of the distribution of these physical variables (visualized as smoke and fire). $N_{c}$ was set to 256 for $\chi$ and $N_{f}$ was set to 256 for $\chi^{R}$ for DBCM method
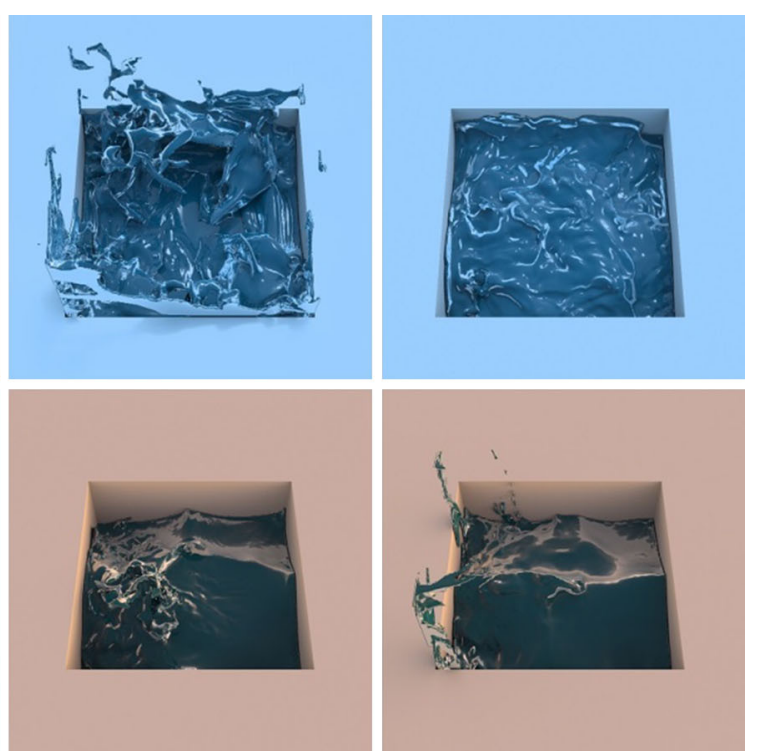

128 and $N_{f}$ was 256 . The bunny model is courtesy of the Stanford 3D Scanning Repository 
Fig. 8 Comparison of semi-Lagrangian advection and our method. Though the level sets were advected under the same velocity field, our method was able to capture many small details of the water surface
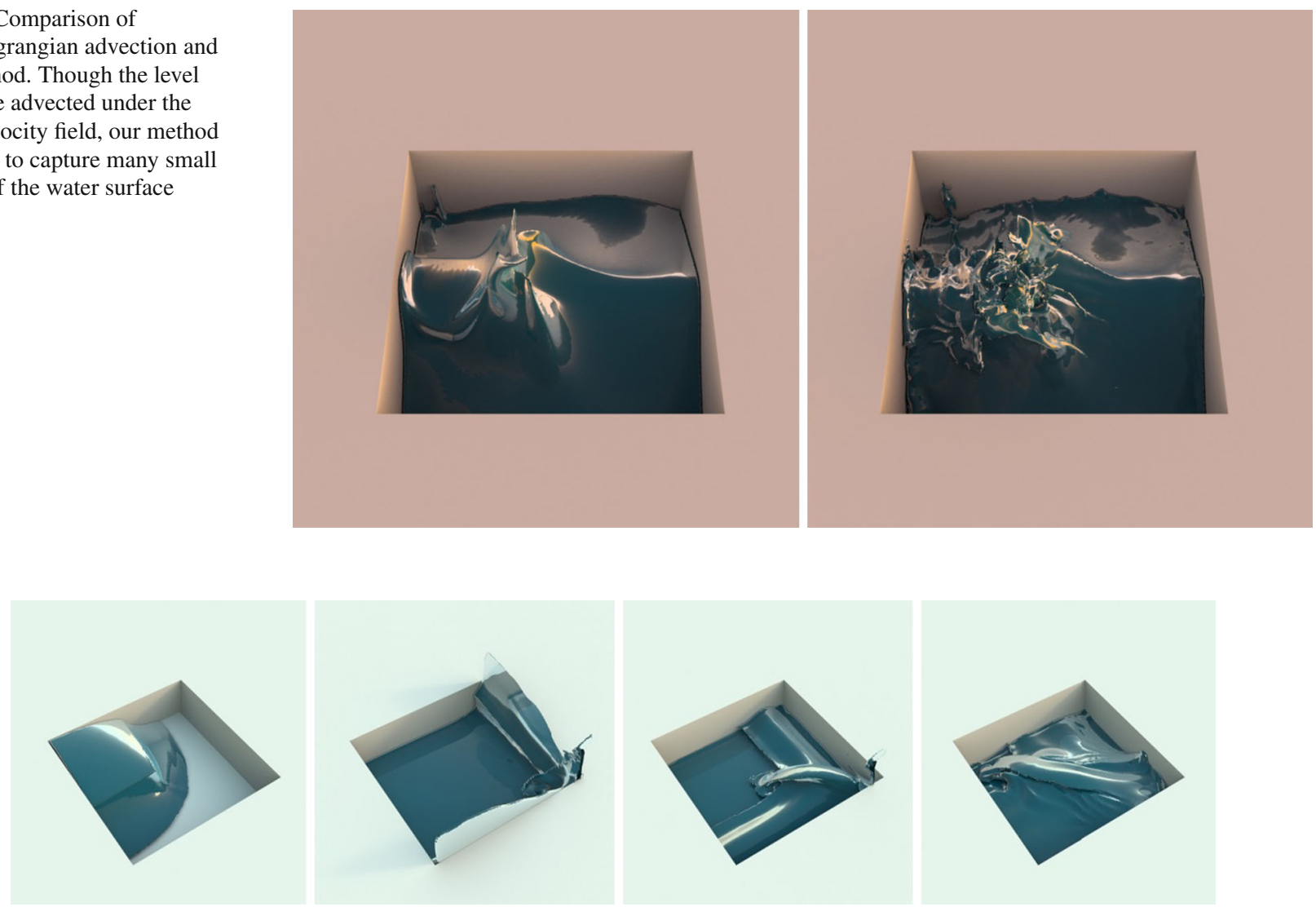

Fig. 9 Dam breaking simulation by the combination of SLC with our DBCM method. Note the thin sheets captured by our method. The resolution of base fluid simulation was $64^{3} . N_{c}$ was 128 and $N_{f}$ was 256 for DBCM method

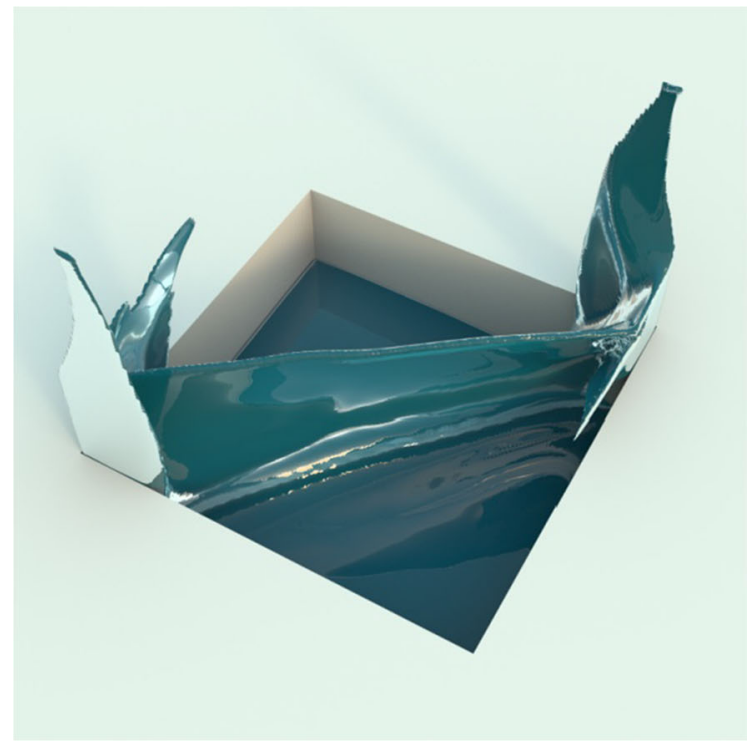

Fig. 10 Double-dam breaking simulation by the combination of SLC with our method. Two columns of water were released to collide with each other to create tall thin sheets and then fell down. Our method

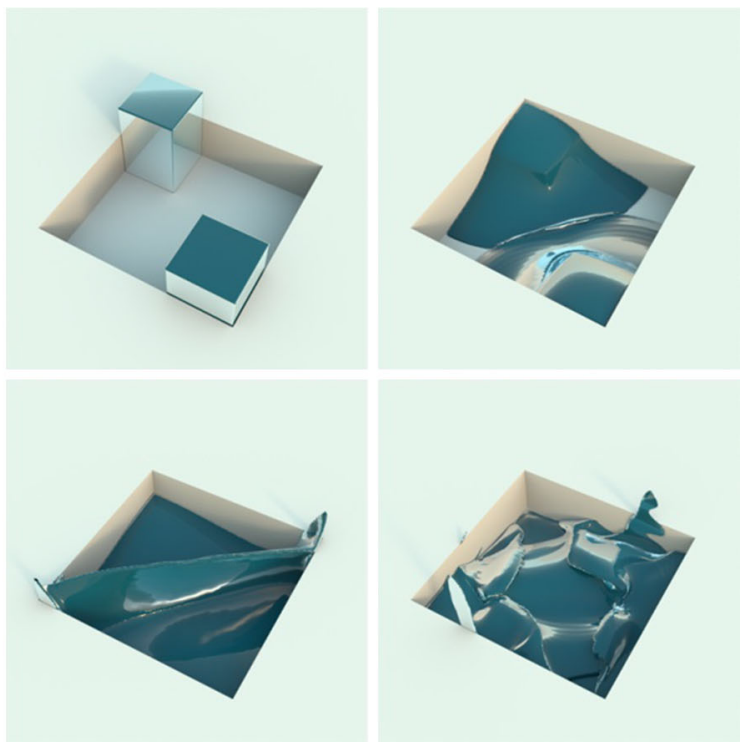

captured such details easily without much effort. The resolution of base fluid simulation was $64^{3} . N_{c}$ was 128 and $N_{f}$ was 256 